\title{
Erythrocebus patas
}

National Cancer Institute

\section{Source}

National Cancer Institute. Erythrocebus patas. NCI Thesaurus. Code C161037.

The diurnal primate species, Erythrocebus patas. 